PSICOLOGÍA

IBEROAMERICANA
Psicología Iberoamericana ISSN: 1405-0943

revista.psicologia@ibero.mx

Universidad Iberoamericana, Ciudad de México México

\title{
La Kaxumpikua elemento sociocultural regidor del comportamiento de los P'urhépecha
}

Navarro Contreras, Gabriela; Correa Romero, Fredi Everardo; Gregorio Cipriano, Fatima

La Kaxumpikua elemento sociocultural regidor del comportamiento de los P'urhépecha

Psicología Iberoamericana, vol. 27, núm. 2, 2019

Universidad Iberoamericana, Ciudad de México, México

Disponible en: http://www.redalyc.org/articulo.oa?id=133962309004 


\title{
La Kaxumpikua elemento sociocultural regidor del comportamiento de los P'urhépecha
}

Kaxumpikua, the sociocultural concept that governs P'urhepecha behavior

\author{
Gabriela Navarro Contreras g.navarro@ugto.mx \\ Universidad de Guanajuato, México \\ http://orcid.org/0000-0003-4744-2351 \\ Fredi Everardo Correa Romero \\ Universidad de Guanajuato, México \\ http://orcid.org/0000-0002-5856-7232 \\ Fatima Gregorio Cipriano \\ El Colegio de Michoacán, México
}

http://orcid.org/0000-0002-8013-7722

Psicología Iberoamericana, vol. 27, núm. 2, 2019

Universidad Iberoamericana, Ciudad de México, México

Recepción: 12 Septiembre 2019 Aprobación: 04 Diciembre 2019

Redalyc: http://www.redalyc.org/ articulo.oa?id=133962309004
Resumen: Uno de los pilares de la sociocultura en la cultura P'urhépecha es la noción de Kaxumpikua, definida de forma conceptual como las normas, reglas y valores por las cuales los miembros de su comunidad rigen su conducta. Sin embargo, la asimilación de los conceptos no es estática, por lo que su comprensión y uso va sufriendo modificaciones y con ello un impacto en la estructura social. El objetivo del estudio fue describir la conceptualización que han asimilado personas pertenecientes a comunidades P'urhépecha del término Kaxumpikua. Para ello se realizó un estudio descriptivo con metodología cualitativa basado en el paradigma de la Teoría Fundamentada, a través de 21 entrevistas semiestructuradas. Entre los resultados más relevantes obtenidos a través de un análisis de contenido asistido por computadora con el programa Atlas.ti 7.5, se encuentra el amplio significado que tiene el concepto entre la comunidad, siendo un baluarte para la integración de la sociedad, en buena medida porque se encuentra ligado con el concepto de respeto hacia los padres, la comunidad y el entorno. Se discute el paralelismo de este concepto con algunas de las premisas histórico-socioculturales de Díaz-Guerrero, así como su utilidad en el desarrollo de escalas culturalmente relevantes.

Palabras clave: cultura, normas, valores, cultura P’urhépecha, Kaxumpikua.

Abstract: One of the pillars of socioculture in the P'urhépecha culture is the notion of Kaxumpikua, which is defined as the norms, rules and values by which members of their community govern their behavior. However, the assimilation of this concept is not a static process and undergoes changes which impact the social structure. For that reason, the objective of this research was to describe the concept of Kaxumpikua in the P'urhépecha communities. For that purpose, a descriptive, qualitative study, drawing on grounded theory, was carried out and 21 semi-structured interviews were conducted. Among the most relevant results, obtained through a computer-assisted content analysis with the Atlas.ti 7.5 program, is that Kaxumpikua represents a bulwark for the integration of society, and is linked to the concept of respect for parents, elders, the community and environment. The parallelism of this concept is discussed in terms of the historical-sociocultural premises of Díaz-Guerrero, as well as its usefulness in the development of culturally relevant scales.

Keywords: culture, Kaxumpikua. 


\section{Introducción}

La cultura es un concepto amplio y complejo que ha sido objeto de estudio desde distintas disciplinas y perspectivas, razón por la cual existen diversas y muy diferentes definiciones. Desde el campo de la psicología transcultural y con un enfoque acorde a la etnopsicología, retomamos el concepto de cultura como el conjunto de actitudes, valores, creencias y conductas compartidas por el grupo de personas, pero diferente para cada individuo y transmitidas de generación en generación (Matsumoto, Kudoh, \& Takeuchi, 1996). Adicionalmente Kroeber y Kluckhohn (1952) explican que la cultura está compuesta de dos elementos, uno subjetivo y otro objetivo, encontrando que las creencias es el aspecto común en ambas definiciones, pues tienen que ver con el proceso de interpretación y asimilación de la realidad social. Con base en estos enfoques, el estudio de elementos culturales es descriptivo, comprensivo para conocer los procesos sociales que vive una comunidad, pero también es la base para el desarrollo de mediciones culturalmente relevantes que permitan buscar elementos en común en diferentes grupos culturales.

En el presente trabajo nos centraremos en el elemento subjetivo de la cultura, definido de forma específica por Matsumoto y Juang (2013) como un sistema único de información y significados, el cual es compartido por un grupo y transmitido a través de las generaciones. Dicho sistema, de acuerdo con los autores previamente citados, permite al grupo satisfacer sus necesidades básicas de supervivencia, buscar la felicidad, el bienestar, así como entender el significado de la vida. Así mismo Díaz-Guerrero (1994) denomina este elemento subjetivo de la cultura como "sociocultura" y lo define como un sistema de premisas "socioculturales" interrelacionadas que norman y gobiernan las ideas, la jerarquización de las relaciones interpersonales y estipulan los papeles sociales que hay que llenar y las reglas de integración de los individuos en tales papeles. En otras palabras, establece dónde, con quién y cómo desempeñar los roles. Desde esta perspectiva se le toma como determinante del comportamiento y de las relaciones interpersonales. Díaz-Loving y Cortés (2011) mencionan, refiriéndose a la tradición etnopsicológica en México y más específicamente a la sociocultura, que la cultura en la cual crecen los individuos brinda tanto los fundamentos como la estructura y las normas de lo que constituye el comportamiento aceptable. Mencionan que "el comportamiento social es dirigido y determinado por la medida en que cada sujeto cree, se adhiere, se dirige e internaliza sus mandatos culturales" (Díaz-Loving \& Cortés, 2011, p. 262).

En esta línea, para la cultura P'urhépecha uno de los pilares subjetivos de su sociocultura es la noción de Kaxumpikua, la cual tradicionalmente ha sido considerada como el eje rector de la conducta de cada persona en la sociedad P'urhépecha. 


\section{La Cultura P'urhépecha}

Los Purépechas habitan en la región norcentral del estado de Michoacán. Esta área se le denomina P'orhépecheo o Purhépecherhu, que significa "lugar donde viven los P'urhé". Abarca aproximadamente el 10\% de los $60000 \mathrm{~km} 2$ que tiene el estado, principalmente en las religiones lacustres y montañosas del centro del estado y en ella habitan más de 200000 personas. Tiene sus orígenes desde épocas muy remotas. El núcleo de la cultura P'urhépecha, refiriéndonos a la geografía nativa de los P'urhépechas, se divide en 4 regiones: Japóndarhu (lugar del lago), Eráxamani (cañada de los 11 pueblos), Juátarisi (meseta), la Ciénega de Zacapu. Es considerada una de las principales culturas mesoamericanas.

La economía de la cultura P'urhépecha tradicionalmente se ha basado en actividades primarias como la agricultura, la pesca, la recolección y la cacería, pero actualmente se han sumado la producción de artesanías, el comercio y el turismo, con lo que se ha diversificado la economía de la región. Estas actividades se sustentan en las familias como unidades para la obtención de ingresos. Políticamente se organizan mediante un consejo de ancianos y la elección democrática de jefaturas. Las comunidades están divididas en barrios que tienen funciones administrativas y ceremoniales. Finalmente, existen autoridades oficiales a nivel federal como el jefe municipal de tenencia, los jueces de registro civil, el consejo de vigilancia, etcétera.

Desde tiempos atrás la denominación de este pueblo indígena ha sido un conflicto, ya que no ha existido consenso de parte de los historiadores sobre su verdadero nombre, sobre si denominarle P'urhépecha o Tarasco. La denominación "tarasco" ha sido utilizada por los historiadores, y es el nombre que al reino y a la lengua de Michoacán se le dio en el tiempo de la conquista (Warren, 2007). La denominación P'urhépecha es más utilizada para referirse a los descendientes de los tarascos (Cortés, 2007). En la época actual se les conoce como P'urhépechas y así también es como estas comunidades se autodenominan.

La religión que predomina y la que es socialmente aceptada es la católica, aunque se practica con una mezcla de creencias autóctonas, pues se cree que los santos forman parte de la vida diaria y que ellos nos ven en todo momento (Van Zantwijk, 1994). Los P'urhépecha conviven bajo dos lenguas el P'urhépecha y el español. El P'urhé es la lengua materna de los P'urhépecha, y aunque la presencia del español es constante, siendo ésta su segunda lengua, es por el $\mathrm{P}^{\prime}$ urhépecha que se comunican, se enseñan todos los conocimientos autóctonos, sus creencias, tradiciones y valores. A pesar de que algunos jóvenes ya no lo hablen, aún lo entienden.

Sin embargo, para los hablantes de esta lengua, la escritura del P'urhépecha no forma parte de la vida cotidiana; muy pocos de ellos la escriben. El español tiene la función de mantenerlos en sintonía con los no P'urhépechas, conocidos como "turhïse". La mayoría de las personas P'urhépecha tienen nociones básicas del español, ya que utilizan las frases o palabras más comunes y las más útiles para su desenvolvimiento fuera de las comunidades. 


\section{La Kaxumpikua}

Adentrándonos en una parte de la cultura subjetiva de los $\mathrm{P}^{\prime}$ urhépechas, nos encontramos un elemento sociocultural indispensable que rige y norma su conducta, y la llaman Kaxumpikua.

Fray Maturino Gilberti traduce el término Kaxumpikua como cortesía o buena crianza (Gilberti, 1990, p. 332). La Kaxumpikua también se traduce al español como cortesía, buenas maneras o buena educación; es decir, designa al que sabe comportarse en la sociedad P'urhépecha de acuerdo con las enseñanzas de los mayores (Jacinto,1998). Otros la conceptualizan como respeto, y entrelazan la Kaxumpikua con la Janhanharhikperata (respeto), manejando a la Kaxumpikua como conducta reverencial, que puede ser traducida como urbanidad, buenos modales o etiqueta, aludiendo a una conducta reverente y reservada hacia los mayores (Padilla, 2000). Sin embargo, la Janhanharhikperata implica el reconocimiento del otro, es una conducta opuesta a la indiferencia, implica la obligación de reconocer la presencia del otro y comunicarle tal reconocimiento (Padilla, 2000).

De acuerdo con Padilla (2000) se podría decir de manera general que la Kaxumpikua se refiere al conjunto de conductas que se expresan visiblemente como señal de respeto hacia el otro y la Kanhanharhikperata es el reconocimiento que se da al otro, el valor que se le asigna al otro y que es visible a través de la conducta de reverencia que se le da (Kaxumpikua).

De acuerdo con Dietz (1999), la Kaxumpikua también se refiere a las relaciones corporales. Éstas entendidas como las maneras de la posición del cuerpo, la distancia que se toma y las gesticulaciones faciales. Según refiere el autor se dan dichas relaciones al estar en interacción con el otro, por lo general de mayor edad o de mayor estatus, además de dictar las normas del buen comportamiento ante la sociedad (Dietz, 1999). Jacinto (1998) menciona que no hay mejor alabanza ni mejor recomendación de un hijo de familia, que el que una persona mayor diga de él que es kaxumpiti, ya que, por el contrario, una persona no-kaxumpiti es aquella que viola las reglas de comportamiento, es aquel que no es recatado.

Todas estas definiciones dan cuenta de un concepto central e integrador en la sociocultura de los P'urhépechas. Llama la atención la diversidad de elementos que la conforman, por lo cual resulta de interés indagar si se mantienen presentes dichos elementos entre los pobladores de la Cañada de los Once Pueblos, así como si aún le dan un sentido dinámico al término Kaxumpikua.

Más importante aún es que de acuerdo con Girola (2005) cuando en las culturas estos valores tradicionales y centrales dejan de tener un significado claro o se abandonan, se corre el riesgo de fomentar conductas anómicas, es decir, conductas fuera de la norma, por lo que se incrementan los problemas como delincuencia, adicciones, suicidios, violencia intrafamiliar, etcétera. Dicho fenómeno ha sido ampliamente documentado en las ciencias sociales por Durkheim (2013), Merton (2002) y, de acuerdo con Girola (2005), la falta de claridad en conceptos 
centrales de la sociocultura es un indicador en América Latina de que una cultura transita hacia posturas más individualistas.

Entonces, dado que todo concepto central para la sociocultura tiene un factor subjetivo que se asimila de forma diversa, nos propusimos mediante el presente estudio cualitativo desde el paradigma de la Teoría Fundamentada describir la conceptualización que se tiene del término Kaxumpikua entre personas pertenecientes a 7 comunidades P'urhépecha (Ichán, Zopoco, Santo Tomás, Tacuro, Carapan, Huáncito y Acachuén), todas de la Cañada de los Once Pueblos del estado de Michoacán.

La importancia de un estudio descriptivo de estas características radica no sólo en conocer un elemento subjetivo de la sociocultura sino, principalmente, asomarse a la importancia y uso del término Kaxumpikua, así como aportar elementos para una medición culturalmente relevante que ayude a detectar necesidades sociales y evitar problemas anómicos en la sociedad.

\section{Método}

El diseño del estudio es transversal, con alcance descriptivo, muestreo intencional no probabilístico, con metodología cualitativa y técnicas de recolección de datos a través de entrevistas semiestructuradas (Kerlinger $\&$ Lee, 2002).

\section{Participantes}

Se entrevistó a 21 personas de cada una de las siguientes comunidades P'urhépecha de la Cañada de los Once Pueblos (3 participantes por comunidad): Carapan, Tacuro, Ichán, Huáncito, Zopoco, Santo Tomas, y Acachuén. Del total de participantes 16 eran hombres y 5 mujeres. El rango de edad fue de los 12 a los 72 años (ver la Tabla 1 ). 
Tabla 1

Caracteristicas de los participantes

Tabla 1

\begin{tabular}{|c|c|c|c|c|c|c|}
\hline $\begin{array}{c}\text { Clave } \\
\text { ID }\end{array}$ & Comunidad & Sexo & Edad & Escolaridad & Ocupación & Generación \\
\hline $1 \mathrm{~A}$ & Ichán & Hombre & 72 & Primaria & Músico & Abuelo \\
\hline $2 \mathrm{~A}$ & Ichán & Hombre & 40 & Secundaria & Músico & Papá \\
\hline $3 \mathrm{~A}$ & Ichán & Hombre & 18 & Preparatoria & Estudiante & Hijo \\
\hline 4B & Zopoco & Hombre & 65 & Primaria & Campesino & Abuelo \\
\hline $5 B$ & Zopoco & Mujer & 40 & Secundaria & Costurera & Mamá \\
\hline $6 \mathrm{~B}$ & Zopoco & Mujer & 14 & Secundaria & Estudiante & Hija \\
\hline $7 \mathrm{C}$ & Santo Tomas & Hombre & 57 & Licenciatura & Maestro & Abuelo \\
\hline $8 C$ & Santo Tomas & Hombre & 36 & Licenciatura & Maestro & Papá \\
\hline $9 \mathrm{C}$ & Santo Tomas & Mujer & 14 & Primaria & Estudiante & Hija \\
\hline $10 \mathrm{D}$ & Tacuro & Mujer & 58 & Primaria & Comerciante & Abuela \\
\hline 11D & Tacuro & Hombre & 42 & Licenciatura & Maestro & Papá \\
\hline $12 \mathrm{D}$ & Tacuro & Hombre & 18 & Preparatoria & Estudiante & Hijo \\
\hline $13 \mathrm{E}$ & Carapan & Hombre & 55 & Secundaria & $\begin{array}{l}\text { Locutor de } \\
\text { radio }\end{array}$ & Abuelo \\
\hline $14 \mathrm{E}$ & Carapan & Mujer & 36 & Secundaria & Ama de casa & Mamá \\
\hline $15 \mathrm{E}$ & Carapan & Hombre & 17 & Preparatoria & Estudiante & Hijo \\
\hline $16 \mathrm{~F}$ & Huáncito & Hombre & 62 & Licenciatura & Profesor & Abuelo \\
\hline $17 \mathrm{~F}$ & Huáncito & Hombre & 36 & Licenciatura & Profesor & Papá \\
\hline $18 \mathrm{~F}$ & Huáncito & Hombre & 12 & Primaria & Estudiante & Hijo \\
\hline $19 G$ & Acachuén & Hombre & 67 & Secundaria & Carnicero & Abuelo \\
\hline $20 \mathrm{G}$ & Acachuén & Hombre & 41 & Preparatoria & Taxista & Papá \\
\hline $21 \mathrm{G}$ & Acachuén & Hombre & 16 & Secundaria & Estudiante & Hijo \\
\hline
\end{tabular}

Nota: Clave ID= Clave de identificación de los participantes.

Nota: Clave ID= Clave de identificación de los participantes.

\section{Técnica de recolección de datos}

La recolección de información se realizó mediante entrevistas semiestructuradas, realizadas en P'urhépecha, que es la lengua materna de los participantes y de una de las autoras. Las entrevistas se grabaron con el consentimiento de los entrevistados siguiendo el protocolo ético para este tipo de estudios (Sociedad Mexicana de Psicología, 2010) y posteriormente se tradujeron y transcribieron al español por una de las investigadoras, que es bilingüe y cuya lengua materna es el P’urhépecha. Los temas sobre los que versó la entrevista fueron: ¿qué es la Kaxumpikua? ¿Por qué es importante la Kaxumpikua? ¿Para qué les sirve la Kaxumpikua? ¿Cómo se enseña la Kaxumpikua? 


\section{Procedimiento}

La recolección de datos se realizó en 3 fases o etapas. Fase 1. Recolección de la información. Al ubicar a los participantes se les explicaba el propósito del estudio y se le preguntó si querían participar. Si la persona accedía se procedía a realizar las entrevistas, las cuales, previo consentimiento, fueron grabadas en una grabadora de voz. La duración de las entrevistas fue de alrededor de 30 minutos. Fase 2. Transcripción y traducción de la información. Después de tener completas las entrevistas, se procedió a transcribirlas y traducirlas al español en el programa Microsoft Word. Las preguntas generales sobre las que versaron las entrevistas fueron: ¿para usted qué es la Kaxumpikua?; ¿por qué es importante la Kaxumpikua?; ¿para qué les sirve la Kaxumpikua?; ¿cómo se enseña la Kaxumpikua?; ¿cómo se puede saber si una persona es Kaxumpite?; ¿crees que la generación de sus nietos sean Kaxumpites?; ¡crees que la generación de tus hijos sean Kaxumpites?; ¿para qué le sirve a la comunidad la Kaxumpikua? Fase 3. Posterior a la captura de la información, los documentos se asignaron al programa Atlas.ti versión $7.5^{\circ}$, donde a partir de un análisis de contenido basado en el paradigma de la teoría fundamentada (Strauss \& Corbin, 1990) se clasificaron las respuestas en códigos emergentes, es decir, se crearon conjuntos de respuestas con contenido similar. Cada conjunto de respuestas se nombró y se estableció como un código cuyo nombre, definición y contenido era independiente del resto de los códigos que fueron emergiendo del análisis. Posteriormente se volvieron a revisar las respuestas que formaban cada código y se verificó su grado de similitud con el resto de las respuestas y se volvieron a revisar los nombres y definiciones de los códigos. Cabe aclarar que, dada la amplitud de la información obtenida en las entrevistas, se decidió analizar en el presente trabajo sólo la parte de la conceptuación y transferencia de la Kaxumpikua. Finalmente, y como lo sugiere Rodríguez, Gil y García (1999), se realizó un microanálisis de contenido para las preguntas seleccionadas, misma que se sintetizó en networks que muestran una descripción esquemática de los códigos. Las relaciones propuestas se basan en el contenido de los códigos emergentes, es decir, el análisis es producto de la interpretación de las respuestas que los participantes aportaron a cada una de las preguntas seleccionadas.

\section{Resultados}

Como se mencionó previamente el análisis de contenido de la información basado en el paradigma de la teoría fundamentada (Strauss \& Corbin, 1990), permitió obtener una serie de códigos emergentes, los cuales se encuentran constituidos por conjuntos de respuestas de más de un participante. La cantidad de respuestas que conforman cada código no es relevante solamente por el número ya que también se toma en consideración la calidad de las respuestas, sin embargo, si aporta una evidencia de la difusión que el concepto tiene entre los entrevistados (ver Tabla 2). 
Tabla 2

Códigos con conjuntos de citas consistentes entre los entrevistados

Tabla 2

Códigos con conjuntos de citas consistentes entre los entrevistados

\begin{tabular}{lc}
\hline Códigos & \multicolumn{2}{c}{$\begin{array}{c}\text { Citas } \\
\text { coincidentes }\end{array}$} & 28 \\
\hline La familia como núcleo para la enseñanza de la Kaxumpikua & 16 \\
La Kaxumpikua como meta valor & \\
La Kaxumpikua como un aprendizaje adquirido por la & 13 \\
comunicación jerárquica & \\
Controversia del papel de la escuela en la enseñanza de la & 10 \\
Kaxumpikua & \\
La Kaxumpikua como una forma de relacionarse en las & 10 \\
comunidades P'urhépecha & 10 \\
Transmisión jerárquica intergeneracional de la Kaxumpikua & 4 \\
La sociedad como vigilante del cumplimiento de la Kaxumpikua & 4 \\
Pérdida de la Kaxumpikua por parte de las nuevas generaciones & 3 \\
El libre albedrío en la adopción de la Kaxumpikua & 2 \\
Formas de aprender la Kaxumpikua &
\end{tabular}

Nota: Se muestran las citas coincidentes entre varios entrevistados.

Nota: Se muestran las citas coincidentes entre varios entrevistados

En ese sentido, son 6 los códigos más importantes: 1. controversia del papel de la escuela en la enseñanza de la Kaxumpikua; 2. el libre albedrío en la adopción de la Kaxumpikua; 3. la familia como núcleo para la enseñanza de la Kaxumpikua; 4. la Kaxumpikua como meta-valor; 5. la Kaxumpikua como un aprendizaje adquirido por la comunicación jerárquica; y 6. la Kaxumpikua como una forma de relacionarse en las comunidades P'urhépecha. A continuación se describen los códigos y se ejemplifican con respuestas de los entrevistados:

Controversia Del Papel De La Escuela en La Enseñanza De La Kaxumpikua

Con base en las respuestas obtenidas hay 2 tipos de ideas entre los entrevistados. Por un lado, que la Kaxumpikua se enseña en la familia y en la escuela, sobre todo entre los participantes de las nuevas generaciones.

"Nuestros padres son quienes nos enseñan y aparte los maestros." (5B, M 40). 
Por otro lado, existe la idea de que el concepto de Kaxumpikua no se puede enseñar en la escuela, dado que es parte de la comunidad y son los padres los que la deben de enseñar junto con los adultos.

"diciéndoles que se porten bien, que no tomen, que saluden a las personas, que no roben, en general se les dice que no deben de hacer, eso se enseña en casa y no en la escuela; en la escuela se enseñan otras cosas." (16F, H 62).

"nuestros abuelos nos enseñan o hasta nuestros bisabuelos que ya tienen más experiencia, o nuestros padres." (6B, M 14).

Esta dualidad es una muestra del dinamismo del concepto y del arraigo que una parte de los entrevistados tiene hacia él y sobre todo la defensa de quién es el responsable de su enseñanza, dando un especial valor a la experiencia.

\section{El libre albedrio en la adopción de la Kaxumpikua}

A pesar de que hay una clara expresión de que la Kaxumpikua se enseña en casa, por los adultos o en la escuela, se acepta que es la persona la que debe abrazar y seguir su enseñanza e incorporarla a su forma de vivir:

"La Kaxumpikua es algo que nosotros los P' urhépecha lo aprendemos a través de la experiencia, y nos lo han enseñado nuestros abuelos o padres, nos decían que hiciéramos bien las cosas." (7C, H 57).

Por lo que no hay una relación de imposición, sino de enseñanza - aprendizaje, de adquisición del concepto, donde la experiencia es un elemento esencial.

"Como lo he dicho, yo trato de enseñarles a mis hijos (la Kaxumpikua) ya es su responsabilidad si lo practican o no.” (12D, H 18).

\section{La familia como núcleo para la enseñanza de la kaxumpikua}

La noción que más menciones tuvo (en ocasiones más de una mención por entrevistado) es que la Kaxumpikua es un concepto que se aprende en familia.

"los responsables de su enseña son los padres, si en la casa no se empieza, entonces, los hijos no van a ser Kaxumpites." (21G, H 16).

En este sentido, los padres y la familia extensa cobran importancia, a veces se menciona al hogar, a los abuelos o a los más grandes como apoyo de la enseñanza de este concepto.

"El respeto nos enseñaban nuestros padres, nuestros abuelos, nuestros antepasados, ellos nos decían que estaba bien, es que anteriormente nos decía que estaba mal, por ejemplo, el mentir." (19G, H 67).

\section{La Kaxumpikua como meta-valor}

Se utiliza el prefijo meta en su acepción epistemológica, es decir, cuando se usa para referirse sobre algo en su propia categoría; en otras palabras, es un valor producido por valores. Y es que la definición que los entrevistados 
hacen de este concepto es diverso, amplio, pero tiene elementos en común:

"La Kaxumpikua la podríamos traducir como un respeto, respeto que se debe de tener hacia ciertas cosas, no simplemente como a personas, el respeto en todos los sentidos." (21G, H 16).

"Se podría traducir como valores, porque allí entran todos los valores." (6B, M 14).

"trata de respetar, trata de cuidar esto y yo lo fui desde niño de alguna manera siguiendo esas reglas de respeto." (8C, H 36).

Como se puede ver la Kaxumpikua da cuenta de valores, donde el respeto hacia los otros es sumamente importante. Adicionalmente, un entrevistado lo definió como "vivir bien" (7C, H 57), lo que habla de que es un valor que hay que poner en práctica en la vida cotidiana.

\section{La Kaxumpikua como un aprendizaje adquirido por la comunicación jerárquica}

En la presente categoría se expresa como además de quién, y dónde se enseña, la forma de transmisión es un elemento importante, pues se comunica por los miembros mayores de la familia a los más jóvenes, pero a través del ejemplo:

"Entonces si tú te comportas bien, con más facilidad a ti también te escuchan y obedecen, pero si no lo eres, es más difícil porque si tu portándote bien no te hacen mucho caso, ahora imagínate, me tacharían de mentiroso, porque yo no enseño con el ejemplo." (19G, H 67).

"En la actualidad esta difícil de enseñarles esas cosas, yo tengo un hijo adolescente, yo trato de platicarle sobre esas cosas para que les muestre respeto a las personas mayores o a los que tienen un cargo." (20G, H 41).

"La Kaxumpikua es algo que nosotros los $\mathrm{P}$ 'urhépecha lo aprendemos a través de la experiencia, y nos lo han enseñado nuestros abuelos o padres, nos decían que hiciéramos bien las cosas." (7C, H 57).

También se nota que la atención, la escucha y la experiencia son importantes, aspecto que tiene sentido al verlo como un valor que se debe poner en práctica a diferencia de un valor idealizado.

\section{La Kaxumpikua como una forma de relacionarse en las comunidades P'urhépecha}

En esta categoría se encuentra contenido que da cuenta sobre lo que es la Kaxumpikua, sin que se haya preguntado de forma directa. Se mencionan ejemplos y conductas.

"en la casa se empieza, darte a respetar en tu familia, pero si tu no respetas a ti tampoco nadie te va a respetar, y así la Kaxumpikua se va perdiendo." (20G, H 41).

"invitar a los padres que platiquen con sus hijos, que no se olviden de darles consejos; es en la casa en donde está la Kaxumpikua, el respeto, y el apoyo." (13E, H 55). 
Es importante mencionar que estos conceptos son comunes entre todos los entrevistados miembros de las comunidades P'urhépecha de la Cañada de los Once Pueblos. Su importancia es tal que constantemente se regresa a su explicación.

En la Figura 1 se integran, en un network creado con ayuda del programa Atlas.ti 7.5, donde se muestran varias citas que dan cuenta de la complejidad y amplitud del concepto de Kaxumpikua como meta valor y su utilización en la cultura P'urhépecha. En dicha figura se puede apreciar que, como valor, se utilizan respuestas como humilde, ser amable, vivir bien, tolerar; y del lado izquierdo se agrupó una serie de respuestas que dan cuenta del respeto. Dicho elemento es tan recurrente que se ligan varias respuestas, algunas de ellas ya correspondientes al código de la Kaxumpikua como forma de relacionarse, donde el respeto es un elemento importante para mantener la estructura familiar y social. En las diversas respuestas se puede observar que la persona debe integrarse, pero no a través del conformismo, sino a través de una interacción constante que toma como referente a los padres, abuelos y en ocasiones a los antepasados lejanos. Algunas citas dan cuenta de que es un proceso que se aprende por la observación y que debe practicarse bajo la supervisión de los más experimentados.

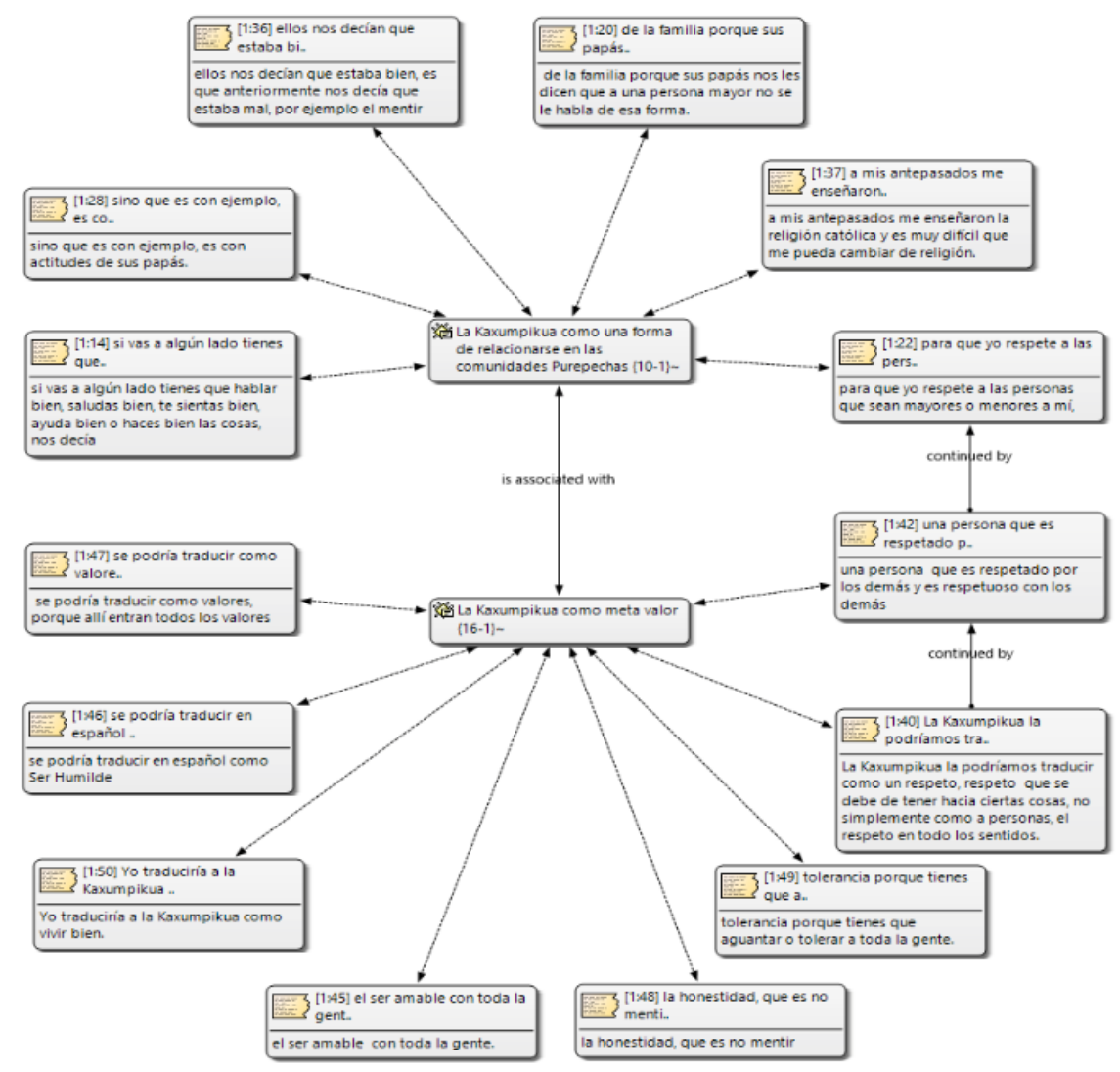

Figura 1. Amplitud y complejidad del concepto de Kaxumpikua

Figura 1.

Amplitud y complejidad del concepto de Kaxumpikua 
En la Figura 2, se presenta otra network realizada con ayuda del programa Atlas.ti 7.5, donde se integran varias categorías para dar cuenta del proceso de transmisión y enseñanza de la Kaxumpikua. Estas categorías tienen como elemento base la familia, pero se complementa con la transmisión jerárquica de toda la sociedad, así como de la escuela.

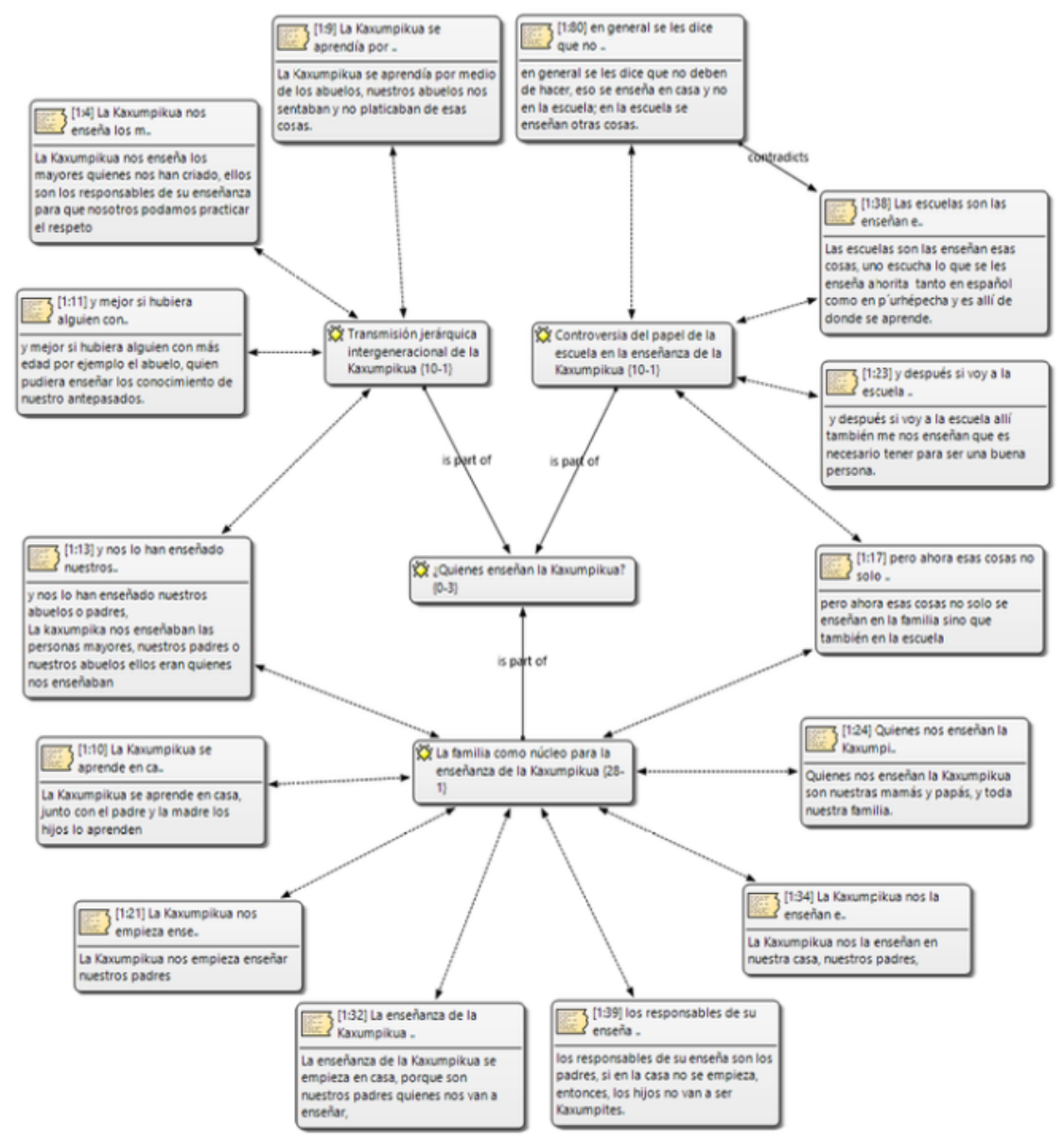

Figura 2. Forma de transmisión de la Kaxumpikua

\section{Figura 2.}

Forma de transmisión de la Kaxumpikua

En las mismas se puede notar que existen citas contradictorias sobre el papel que juega la escuela, pues mientras los mayores opinan que en la escuela no se puede enseñar la Kaxumpikua, los padres y los adolescentes mencionan que ahí también se les enseña.

\section{Discusión}

Como puede observarse en el análisis de contenido realizado, el concepto Kaxumpikua es de gran relevancia para los entrevistados de las comunidades P'urhépecha participantes. Se puede decir que dicho concepto constituye el eje rector del comportamiento en un sentido ecológico ya que tiene influencia tanto a nivel individual como familiar, social e incluso con el entorno ambiental para estas comunidades, a pesar de su complejidad y diversidad. De acuerdo con Mejía-Arauz, Keyser y 
Correa-Chávez (2013), la Kaxumpikua es uno de esos elementos que en culturas indígenas son enseñados y socializados para que la persona pueda emerger, brotar o despertar su expresión como miembro del grupo social. En el caso particular de los P'urhépecha, esa gradual emergencia es resultado del saber comportarse en sociedad de acuerdo con la enseñanza de los mayores.

Resulta relevante hacer notar que el concepto de Kaxumpikua presenta similitudes con las premisas histórico-socioculturales de Díaz-Guerrero (1955), ya que de la misma manera que estas últimas, podemos considerar que la Kaxumpikua es una guía válida dentro de la familia, el grupo, la sociedad y las superestructuras institucionales de las comunidades P'urhépecha. De forma similar, la Kaxumpikua dirige las cosmovisiones responsables del desarrollo de la personalidad, las principales metas de la vida, la forma de enfrentarla, la percepción de la humanidad, etcétera (Díaz-Loving \& Cortés, 2011). En este sentido la Kaxumpikua forma parte de los saberes que se transmiten de generación en generación a nivel familiar o comunitario como un eje de formación comunitaria (Caballero, 2002).

De acuerdo con Díaz-Guerreo y Peck (1967) la sociedad mexicana está construida sobre una estricta estructura jerárquica basada en el respeto hacia aquellos que están más arriba en la jerarquía social, en particular los padres, los mayores y los familiares. La Kaxumpikua guarda cierto paralelismo con la importancia del respeto que plantean dichos autores, sin embargo, la jerarquía en este caso no se observa de la misma manera. Aunque una de las formas del respeto se presente en cierta como deferencia hacia la autoridad y el estatus de jerarquía, la cual se ha atribuido más a la colonización y a las interacciones dentro de países latinoamericanos con marcadas jerarquías socioeconómicas (Rubalcaba, Rogoff, Lopéz, Correa-Chavéz, \& Gutierrez, 2015), en las comunidades indígenas se puede observar un sistema de valores más horizontal. Con base en las descripciones analizadas entre las comunidades Purépecha aquí estudiadas, el respeto no se sustenta en el temor sino en el bien común, el ser Kaxumpite es relevante para la persona, pero también para la familia y para la sociedad como un todo indivisible. En este sentido, el no incorporar la Kaxumpikua a la vida cotidiana, se convierte en un asunto colectivo basado en una responsabilidad mutua. De tal manera que existe una corresponsabilidad del seguimiento de este valor, no en un sentido punitivo sino del deber.

Lo aquí observado es coincidente con la idea de respeto observado en otros pueblos indígenas como plantea Rubalcaba et al. (2015), esto es, basada en un sistema mutuo de valores. Esta idea de respeto incluye tener en consideración la dirección de los proyectos compartidos por el grupo, y no sólo la consideración de otros individuos como individuos. En este sentido la forma mutua del respecto de acuerdo con los autores (Rubalcaba et al., 2015), incluye asimetrías no jerárquicas en los roles de liderazgo, como en la colaboración entre niños y adultos en la cual el liderazgo es flexible, basado en quién puede hacer las cosas y no en jerarquías rígidas. 
Coincidente con lo observado en las entrevistas, otros estudios han encontrado que la falta de respeto puede ser considerada como un reflejo de la crianza que llevaron a cabo los padres y de la familia en sí (Calzada, Fernández, \& Cortes, 2010).

De acuerdo con García (2015) las familias indígenas Quechuas socializan el respeto a través de prácticas como miradas guía, toques en los hombros, pequeños empujones y guiños. Estas conductas motivan al niño a mostrar respeto, y les recuerdan que ésta es una forma de cuidar o preocuparse por los otros. Entre los Quechuas el respeto puede llevarse a cabo a través de ser considerado con los otros y teniendo una participación mesurada. La educación local de estas comunidades emerge con una orientación hacia la formación de personas respetuosas, las cuales aprenden y se desarrollan mediante la observación en silencio, con ciertas restricciones impuestas por los adultos pero que nunca frustran o inhiben sus capacidades para tomar iniciativa (García, 2015).

Este sentido del deber es justo lo que autores como Durkheim (2013), Merton (2002) y Girola (2005) mencionan como el aspecto más relevante de cohesión, que ayuda a evitar las conductas anómicas en comunidades tradicionales.

Desde un enfoque transcultural y de la tradición etnopsicológica seguida en psicología social a partir de las investigaciones de DiazGuerrero (1955), los trabajos de corte cualitativo tienen una amplia riqueza en sí mismos pero, sobre todo, se complementan con los estudios transculturales de tipo cuantitativo. Lo antes planteado es posible debido a que permiten profundizar en la comprensión de los conceptos, para lograr que sean realmente culturalmente relevantes. Ejemplo de lo anterior es el trabajo de Gregorio-Cipriano y Navarro-Contreras (2017), donde se comparan los patrones de respuesta a la escala de premisas histórico-socioculturares (Díaz-Guerrero \& Iscoe, 1984) en español y su adaptación al P'urhépecha en estudiantes bilingües, donde a pesar de que los participantes tenían dominio en ambas lenguas, se encontraron diferencias estadísticamente significativas en los patrones de respuesta a las escalas, obteniendo puntajes mayores en la escala en P'urhépecha, lo cual podría comprometer la precisión de evaluación en el grupo meta.

Entre las limitaciones del estudio se puede mencionar que, por cuestiones analítico-descriptivas focalizadas en el significado de la Kaxumpikua, no se consideró profundizar en los significados para hombres y mujeres ni diferentes grupos de edad, etcétera. Otra limitación fue que el estudio se concentró en una sola técnica de recolección de datos. En futuros estudios la inclusión de grupos de discusión, observación participante, etcétera, permitirán profundizar más en los análisis descriptivos. Finalmente, se sugiere para futuras investigaciones en el tema un análisis desde los principios de la psicología comunitaria, el cual podría permitir una mayor comprensión de los significados y usos de la Kaxumpikua. 


\section{Conclusión}

El objetivo de la investigación fue describir la conceptualización que se tiene del término Kaxumpikua entre personas pertenecientes a comunidades P'urhépecha de la Cañada de los Once Pueblos del estado de Michoacán. Luego de la recolección, captura, selección de las respuestas más relevantes, clasificación en códigos emergentes que fueron integrados en dos redes descriptivo-sintéticas, podemos concluir que la Kaxumpikua tiene un significado más amplio del que académicamente se refieren los textos, pues incluye una serie de valores que giran en torno al respeto de uno mismo, de la familia, la comunidad y el medio ambiente.

Esta complejidad es resultado de la existencia de variaciones culturales, debidas a que las actividades de las personas van cambiando y se realizan en contextos diferentes a lo largo del tiempo, por lo que diversas generaciones terminan conviviendo alrededor de un elemento cultural, que fue educado, socializado y asimilado de forma diferente y en un contexto distinto (Mejía-Arauz et al., 2013).

De acuerdo al análisis de contenido realizado, la Kaxumpikua guarda cierto paralelismo con las premisas histórico-socioculturales de DíazGuerrero (1955) como elemento integrador de la sociocultura. Sin embargo, a diferencia de aquellas premisas que caracterizan al mexicano, la Kaxumpikua guarda características propias de los grupos originarios, dado que como valor no es individual ni impuesto, por el contrario, es colectivo y asimilado a través del ejemplo y la práctica. Lo cual concuerda con Mosier y Rogoff (2003), quienes concluyen que entre muchos grupos indígenas el modelo cultural que prioriza tanto la responsabilidad como el respeto es la libertad de elección y no la imposición.

Queda pendiente para futuras investigaciones analizar algunas funciones de la Kaxumpikua como premisa sociocultural, por ejemplo, su papel en la distinción de roles de género o laborales, así como profundizar en las nuevas formas de trasmisión de este meta-valor, debido a la incorporación de las tecnologías en la vida cotidiana de las comunidades.

Entre los alcances del estudio se logró profundizar en la descripción de la Kaxumpikua, así como en sus diversas formas de transmisión. Los elementos encontrados se corresponden con los descritos por MejíaArauz et al. (2013) para determinar la forma como se socializan las prácticas colaborativas en comunidades P'urhépecha. De acuerdo con los autores, las generaciones más longevas tuvieron actividades colaborativas en el ambiente familiar y dichas actividades estaban ligadas al trabajo, mientras que las nuevas generaciones realizan actividades colaborativas en la escuela y se encuentran orientadas al conocimiento y la preservación de las tradiciones.

Desde un enfoque transcultural, indagaciones como la presentada aquí coadyuva en la identificación de patrones culturales, lenguaje predominante y procesos de significación como lo propone Reyes Lagunes (2011), para evaluar de forma sensible las necesidades sociales de grupos culturales específicos, como aquellos que pertenecen a las comunidades P'urhépecha. 
Finalmente, este tipo de estudios son la base para el desarrollo de políticas públicas que atiendan las necesidades sociales específicas de las comunidades. En ese sentido, Gembe-Sánchez (2016) menciona que se busca que toda investigación no sólo sea una fuente de conocimiento, sino la base del cambio social, pero antes es necesario elaborar y conocer la historia que conforma una comunidad. Lo anterior con la finalidad de que las intervenciones tengan un carácter crítico, evitando la implementación inmediata de cursos o talleres que busquen otorgar conocimientos a la población sin un análisis profundo del contexto de los grupos en estudio.

\section{Referencias}

Caballero, J. J. (2002). Educación y cultura: Formación comunitaria en Tlazoyaltepec y Huitepec, Oaxaca. CDMX, México: CIESAS.

Calzada, E. J., Fernandez, Y., \& Cortes, D. E. (2010). Incorporating the cultural value of respeto into a framework of Latino parenting. Cultural Diversity and Ethnic Minority Psychology, 16(1), 77-86. https://doi.org/10.1037/a 0016071

Cortés, J. C. (2007). Historia de dos nombres: tarascos y P'urhépechas. En P. Márquez (Ed.), ¿Tarascos o P'urhépecha? Voces sobre antiguas y nuevas cuestiones entorno al gentilicio michoacano (pp. 53- 65). CDMX, México: Colección $\mathrm{Kw}^{\prime}$ aniskuyarhani.

Díaz-Guerrero, R. (1955). Neurosis and the Mexican family structure. American Journal of Psychiatry, 112(6), 411-417. https://doi.org/10.1176/ajp.112. 6.411

Díaz-Guerrero, R. (1994). Psicología del mexicano. CMDX, México: Trillas.

Díaz-Guerrero, R., \& Iscoe, I. (1984). El impacto de la cultura iberoamericana tradicional y del stress económico sobre la salud mental y física: Instrumentación y potencial para la investigación trascultural. Revista Latinoamericana de Psicología, 16(2), 167-211.

Díaz-Guerrero, R., \& Peck, R. F. (1967). Estilo de confrontación y aprovechamiento: Un programa de investigación. Revista Interamericana de Psicologia, 1 (2), 127-136. https://doi.org/10.30849/rip/ijp.v1i2.438

Díaz-Loving, R., \& Cortés, M. E. (2011). XI Etnopsicología: ¿Ciencia nova o refundación de la psicología? En A. C. Domínguez Espinosa (Ed.), Lecturas introductorias a la psicología cultural, transcultural y etnopsicología. CDMX, México: Universidad Iberoamericana.

Dietz, G. (1999). La comunidad P'urhépecha en nuestra fuerza: etnicidad, cultura y región en un movimiento indigena en Michoacán, México. Quito, Ecuador: Abya Yala.

Durkheim, E. (2013). El suicidio [The suicide]. CDMX, México: Colofón.

García, F. A. (2015). Respect and autonomy in children's observation and participation in adults' activities. Advances in Child Development and Behavior, 49, 137-151. https://doi.org/10.1016/bs.acdb.2015.10.003

Gembe-Sánchez, M. A. (2016). Re-configuraciones de género en un pueblo urbanizado de la meseta purhépecha (Tesis doctoral). El Colegio de Michoacán, México.

Gilberti, M. (1990). Vocabulario en lengua de Mechuacan. CDMX, México: Centro de Estudios de Historia de México. 
Girola, L. (2005). Anomia e individualismo. Barcelona, España: Anthropos.

Gregorio-Cipriano, F., \& Navarro-Contreras, G. (2017). Premisas Históricosocioculturales en adolescentes bilingües. Diferencias entre los patrones de respuesta al test en español y en P'urhépecha. Revista Educación y Desarrollo, 41, 61-70.

Jacinto, A. (1998). Mitología y modernización. Michoacán, México: Colegio de Michoacán.

Kerlinger, F. N., \& Lee, H. B. (2002). Investigación del comportamiento. Métodos de investigación en ciencias sociales ( $4^{a}$ ed.). CDMX, México: McGrawHill.

Kroeber, A., \& Kluckhohn, C. (1952). Culture: A critical review of concepts and definitions. Cambridge, MA, Estados Unidos: Peabody Museum.

Matsumoto, D., \& Juang, L. (2013). Culture and psychology. Wadsworth, OH, Estados Unidos: Cengage Learning.

Matsumoto, D., Kudoh, T., \& Takeuchi, S. (1996). Changing patterns of individualism and collectivism in the United States and Japan. Culture \& Psychology, 2(1), 77-107. https://doi.org/10.1177\%2F1354067X962100 5

Mejía-Arauz, R., Keyser O, U., \& Correa-Chávez, M. (2013). Transformaciones culturales y generacionales en la participación colaborativa de niñas y niños de una comunidad P urhépecha. Revista Mexicana De Investigación Educativa, 18(59), 1019-1045.

Merton, R. (2002). Teoría y estructura social. CMDX, México: FCE.

Mosier, C., \& Rogoff (2003). Privileged treatment of toddlers: Cultural aspects of individual choice and responsability. Developmental Psychology, 39(6), 1047-1060. https://doi.org/10.1037/0012-1649.39.6.1047

Padilla, M. (2000). Ciclo festivo y orden ceremonial: Sistema de cargos religiosos en San Pedro Ocumicho. Michoacán, México: Colegio de Michoacán.

Reyes Lagunes, I. (2011). XII Conceptuación y desarrollo de la etnopsicometría en México. En A. C. Domínguez Espinosa (Ed.),Lecturas introductorias a la psicología cultural, transcultural y etnopsicología. CDMX, México: Universidad Iberoamericana.

Rodríguez, G., Gil, J., \& García, E. (1999). Metodología de la investigación cualitativa. Málaga, España: Ediciones Aljibe.

Rubalcaba, O., Rogoff, B., Lopéz, A., Correa-Chavéz, M., \& Gutierrez, K., (2015) Chapter eight- Children's avoidance of interrupting others' activities in requesting help: Cultural aspects of considerateness. Advances in Child Development and Behavior, 49, 185-205. https://doi.org/10.101 6/bs.acdb.2015.10.005

Sociedad Mexicana de Psicología. (2010). Código ético del psicólogo (5a ed.). CDMX, México: Trillas.

Strauss, A. L., \& Corbin, J. M. (1990). Basics of qualitative research: Grounded theory procedures and techniques. Newbury Park, CA, Estados Unidos: Sage Publications.

Van Zantwijk, R. (1994). Los servidores de los santos: La identidad social y cultural de una comunidad tarasca en México. CDMX, México: INI-SEP.

Warren, J. B. (2007). Algunas consideraciones histórico-lexicográficas. En P. Márquez (Ed.), ¿Tarascos o P'urhépecha? Voces sobre antiguas y nuevas 
Gabriela Navarro Contreras, et al. La Kaxumpikua elemento sociocultural regidor del comportamiento de los P'urhépecha

cuestiones entorno al gentilicio michoacano (pp. 41-51). CDMX, México: Colección Kw'aniskuyarhani. 